\title{
An Adaptive Video Coding Control Scheme for Real-Time MPEG Applications
}

\author{
Shih-Chang Hsia \\ Department of Computer and Communication Engineering, National Kaohsiung First University of Science and Technology, \\ Kaohsiung 824, Taiwan \\ Email: hsia@ccms.nkfust.edu.tw
}

Received 27 February 2002 and in revised form 16 September 2002

This paper proposes a new rate control scheme to increase the coding efficiency for MPEG systems. Instead of using a static group of picture (GOP) structure, we present an adaptive GOP structure that uses more P- and B-frame coding, while the temporal correlation among the video frames maintains high. When there is a scene change, we immediately insert intramode coding to reduce the prediction error. Moreover, an enhanced prediction frame is used to improve the coding quality in the adaptive GOP. This rate control algorithm can both achieve better coding efficiency and solve the scene change problem. Even if the coding bit rate is over the predefined level, this coding scheme does not require re-encoding for real-time systems. Simulations demonstrate that our proposed algorithm can achieve better quality than TM5, and satisfactory reliability for detecting scene changes.

Keywords and phrases: control strategy, MPEG, rate control, scene change, temporal correlation.

\section{INTRODUCTION}

Recently, the video coding systems have been widely applied to digital TV, video conferencing, multimedia systems, and so forth, primarily, in order to reduce the bit rates $[1,2,3]$. It is well known that most coding techniques will generate variable bit rates in various video sequences. To transmit the variable rate bit stream over a fixed rate channel, a channel buffer is required. Therefore, the main purpose of the rate control algorithm is to prevent the buffer from overflowing and underflowing and to generate a constant bit rate for targets. To regulate the fluctuation of the coding rate, we need to allocate the compressed bit of each frame by choosing a suitable quantization parameter for each macroblock. The fundamental buffer control strategy adjusts the quantizer scale according to the level of buffer utilization $[4,5,6]$. When the buffer utilization is high, the quantization level should be increased accordingly.

In a practical MPEG system, the picture type is selected from Intra, Predict or Bidirectional frames [7]. Moreover, there are many choices for macroblocks coding, including the intraframe code, the interframe code by motion compensation, or simply a replica from the previous frame. The selection of quantization scale, coding mode, and picture type will decide the coding bit rate, and consequently affect the coding quality. Due to the extremely high complexity of the optimal coding, various suboptimal solutions have been proposed $[8,9,10]$. Generally, the image quality is improved about $2 \mathrm{~dB}$ compared with TM5 method [11]. Based on the model of rate distortion curve, the computation load becomes very high. In addition, if the coding result is not satisfactory, re-encoding procedures are required in these approaches. Because this re-encoding process will increase the computational time, it is not desirable for real-time applications.

In this study, a novel coding strategy is proposed to improve the coding efficiency, especially for real-time applications. Our method can decide the coding parameters at once and avoid the need for re-encoding procedures even if the coding bit rate is over the predefined maximum level or a scene change is detected. This paper is organized as follows. An adaptive control strategy is presented in Section 2, experimental results are described in Section 3, and conclusions are given in Section 4.

\section{AN ADAPTIVE CODING CONTROL ALGORITHM}

For video coding systems, first-in first-out (FIFO) memory is generally used to regulate the fluctuation of the coding rate. A basic control structure is shown in Figure 1. As the coding procedure continues, the current FIFO occupation becomes

$$
\mathrm{FIFO}_{\text {current }}=\mathrm{FIFO}_{\text {previous }}+\left(\text { Coding }_{\text {bit }}-\text { Target }_{\text {bit }}\right),
$$

where Coding $_{\text {bit }}$ is the current coding result and Target bit $_{\text {it }}$ is the constant output rate. Since the coding bit rate may be larger or smaller than the target bit rate, a FIFO memory is employed as a regulator to dynamically balance the 


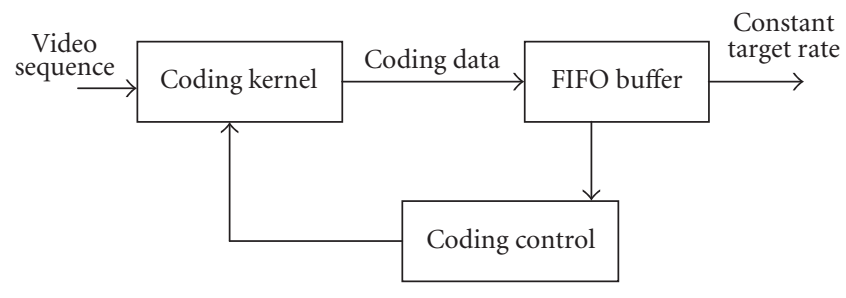

FIgURE 1: The basic coding control scheme.

coding bit rate and the target bit rate. Because the memory size is limited, we need to adjust the quantization level to avoid the buffer to overflow or underflow. In MPEG coding systems, the fixed group of picture (GOP) structure is IBBPBBPBBPBBI, where the I-frame is the basic reference for $\mathrm{P}$ - or B-frame coding. P-frame coding uses the motion prediction from the I-frame or the previous $\mathrm{P}$-frame, and Bframe coding employs the bidirectional prediction between the neighboring I-frame and P-frame, or between two Pframes. Therefore, the total coding bit rate for one GOP is then the sum of the coding bits of each frame, which is

$$
\mathrm{GOP}_{\text {bit rate }}=\mathrm{I}_{\mathrm{bit}}+\mathrm{P}_{\mathrm{bit}}+\mathrm{B}_{\mathrm{bit}} \text {, }
$$

where $\mathrm{I}_{\mathrm{bit}}, \mathrm{P}_{\mathrm{bit}}$, and $\mathrm{B}_{\mathrm{bit}}$ are the coding bits for the I-frame, P-frame, and B-frame, respectively.

\subsection{A new adaptive GOP structure}

When the static GOP structure is used, the coding efficiency of its $\mathrm{P}$ - or B-frames becomes poor for low correlation sequences due to high prediction errors. An extreme case is that if the video sequence changes suddenly, the coded image may produce serious distortions. On the other hand, while the temporal correlation among the video frames is high, we can obtain better performance by applying more P- and Bframes coding. Hence the coding quality will be much better since the motion compensation from the previous frame is done. This is particularly effective for low motion sequences. One of the effective compensation methods is the adaptive GOP (AGOP), where the structure is dynamically modified according to the temporal correlation between interframes.

The AGOP concepts are proposed as follows. First, the Pand B-frames are continuously coded by the prediction mode until one of the following conditions occurs:

(i) if the buffer utilization is very low, then the I-frame will be coded to avoid the buffer underflowing;

(ii) if the video sequence changes suddenly, that is, $\mathrm{P}(n)_{\text {bit }} \gg \mathrm{P}(n-1)_{\text {bit }}$ is detected, where $\mathrm{P}(i)_{\text {bit }}$ is the coding bit rate for the $i$ th $\mathrm{P}$-frame, then we re-encode the $n$th frame using an I-frame coding rather than a P-frame coding;

(iii) if the accumulated error gradually becomes high such that

$$
\mathrm{P}(n)_{\text {bit }} \gg \sum_{k=-m}^{-1} \frac{\mathrm{P}(n+k)_{\text {bit }}}{m},
$$

the current P-frame coding rate is higher than the averaged bit rate of the previous $m$ frames and over a predefined threshold, then the $n$th frame uses an I-frame coding.

In the above processing, the GOP structure is adaptively changed in accordance with the temporal correlation of the previous frames. If the intervening frames have high correlation, we use more prediction coding to reduce the temporal redundancy until the accumulated error becomes too large or a scene change is detected. When video sequences go on, the scene change point may be at the I-, B-, or P-frames. If the scene change is at the I-frame, the reference memory is reset by the I-frame itself, and so there will be no problems for the next P- or B-frame prediction. Since the B-frame has bidirectional prediction, there are no serious errors when the scene change occurs at the B-frame. However, if the scene change occurs on a P-frame, the predicted error will be high due to the lack of temporal correlation. Then the predicted error will accumulate to the next frame coding and the coding performance thus degrades seriously. It is a direct method that we can re-encode the current frame using an I-frame coding for the off-line system when a scene change is detected or the temporal correlation becomes very low. However, we also aim to reduce the processing time as much as possible for the requirements of real-time applications.

For real-time processing requirements, we monitor the coding condition using the slice base in the MPEG system. First, let $N$ be the number of slices used in the coding system. The first $N$ slices bit rate (slice furrent $_{\text {first }}$ ) of the current frame is then compared with the first $N$ slices (slice previous $_{\text {first }}$ ) of the previous frame. In addition, let $\mathrm{Q}_{\text {current }}^{\text {first }}$ and $\mathrm{Q}_{\text {previous }}^{\text {first }}$ denote the averaged quantization scales for the first $N$ slices of the current and the previous frames, respectively. If the averaged coding bit rates of the $N$ slices for the adjacent frames have changed drastically, that is,

$$
\mathrm{Q}_{\text {current }}^{\text {first }} \times\left(\frac{\text { slice }_{\text {current }}^{\text {first }}}{N}\right) \gg \mathrm{Q}_{\text {previous }}^{\text {first }} \times\left(\frac{\text { slice }_{\text {previous }}^{\text {first }}}{N}\right)
$$

indicating that a scene change has been detected between the current frame and the previous one, then a new intracoding is introduced to process the rest of the current frame. The same intracoding is then used for the first $N$ slices of the next frame and its remaining slices return to use the prediction coding. Figure 2 shows the detailed frame coding with a scene change. The comparison begins only when both frames have P-coding in their first $N$ slices, and the new intracoding is again introduced when another drastic change has been detected. Our scheme is hence efficient and fast to satisfy the needs of real-time processing. Furthermore, in our experiments, the number of $N$ is not fixed. The first slice coding rate is checked, and the scene change is found if the coding rate of the current frame is the triple of the previous one in (4). We immediately encode I-mode for the next slices. Otherwise, the first two slices are checked again. With this procedure, we check the averaged coding bits from the first $N$ slices to the whole frame. 


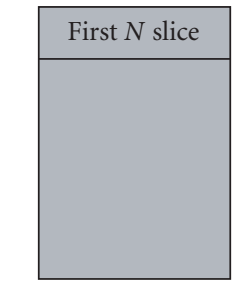

Previous frame $n-1$

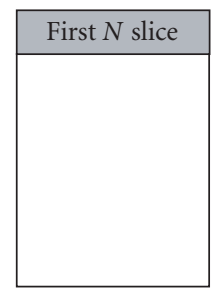

Current frame $n$

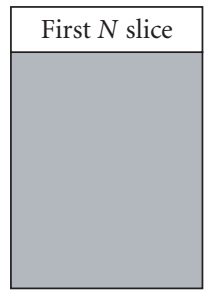

Next frame $n+1$
Scene change

1-frame coding

Predict coding

FIGURE 2: The frame coding as scene change between $(n-1)$ th and $n$th frames.

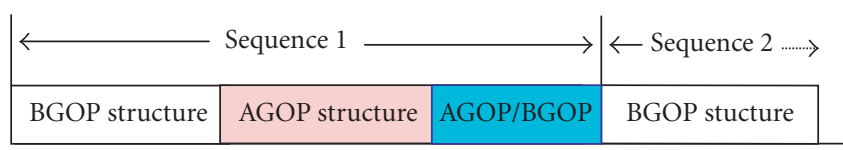

FIgUre 3: The proposed adaptive GOP structure.

Based on this concept, a new AGOP structure is presented in Figure 3. First, the basic GOP (BGOP) structure is employed, consisting of one I-frame, three P-frames, and eight $\mathrm{B}$-frames, where the frame order is the same as the conventional GOP structure for MPEG systems. Next, an AGOP structure is applied, whose length depends on the temporal correlation. Consequently, its length will be considerably shortened if a scene change is detected. In order to enhance the advantage of our new coding scheme, there is no I-frame used in the AGOP structure. We also adopt 12 frames as a coding unit to keep bit rate balancing. The sequence order is then

$$
\mathrm{P}_{\mathrm{e}} \text { BВРВBРВBРВBР }{ }_{\mathrm{e}} \text { BВРВB..., }
$$

where $\mathrm{P}_{\mathrm{e}}$ is an enhanced $\mathrm{P}$-frame with a higher coding bit rate than that of a normal P-frame. We use a $\mathrm{P}_{\mathrm{e}}$-frame rather than an I-frame for high-correlated video sequences in order to reduce the temporal redundancy and the coding bit rate. Hence, the total coding efficiency is increased due to this motion compensation. The AGOP coding scheme ends when a scene change is detected or the accumulated error becomes too large, and then the coding procedure begins another BGOP processing.

It is important to note that for AGOP coding, if the correlation of local blocks is very low between two continuous frames in one sequence, high prediction errors will not only occur in the current block but also will be transferred to the next predicted block. To overcome this drawback, we employ an intrablock coding instead of the interblock coding for low correlation blocks in local areas. The following criterion can determine whether or not the current coding block uses an intrablock coding for P- or B-frames. If the mean absolute difference (MAD) [12] from the result of motion estimation is very large, which implies that the predicted error is very serious, then an I-block coding is employed to reduce the predicted error. The coding mode for a macroblock can be determined by

$$
\begin{aligned}
& \text { if } \mathrm{MAD}<\mathrm{Th}_{0}, \mathrm{MV}=0 \text {, then inter (skip) mode } \\
& \text { else if } \mathrm{Th}_{0}<\mathrm{MAD}<\mathrm{Th}_{1} \text {, then inter (MC+DCT) mode } \\
& \text { else if } \mathrm{MAD}>\mathrm{Th}_{1}, \mathrm{MV} \neq 0 \text {, then intramode, }
\end{aligned}
$$

where thresholds were selected such that $\mathrm{Th}_{1}>\mathrm{Th}_{0}$ is always used. If the MAD of the motion estimation is very low and the motion vector (MV) is zero, this implies that the current block is almost the same as the referenced one. Then the referenced block can be duplicated instead of using the current block coding, so this coding block is assigned as inter (skip) mode. However, if the MAD result of the motion estimation is large, we switch from intermode to intramode to avoid high prediction errors. For fast and instantaneous real-time processing, it is necessary to evaluate the block correlation based on motion estimations first. So the coding mode for the macroblock will be selected from either the intramode or the intermode to achieve better coding quality for each local block.

\subsection{The coding bit rate budget}

\subsubsection{For BGOP structure}

First, we estimate the bit rate for the I-frame coding. Since the I-frame is the basic reference frame, its coding error would be accumulated and propagated to the next P- and $B$-frames. To reduce the prediction error, we must appoint higher a bit rate for the I-frame coding. In any case, the coding bit rate of an I-frame depends on the target rate and the frame rate of the system. Therefore, the bit rate for the Iframe must be constrained in a range of

$$
\frac{\text { Target Rate }}{\text { Frame Rate }} \times \mathrm{IR}_{\mathrm{H}} \geq \mathrm{I}_{\text {bit }} \geq \frac{\text { Target Rate }}{\text { Frame Rate }} \times \mathrm{IR}_{\mathrm{L}},
$$

where $I R_{H}$ and $I R_{L}$ denote the maximum and minimum factors, respectively, which were determined by the buffer status of the system. As the buffer utilization is high, the coding bit rate will be reduced accordingly. In order to control the bit rate in the constrained range, the quantization level for the Iframe is adaptively adjusted dependent on both the previous coding results and the buffer status.

The coding status of the system is monitored by a slicebase method as follows. An initial quantization level is chosen for the first slice coding as

$$
\mathrm{Q}_{0}^{\mathrm{I}}=\frac{\mathrm{Q}_{\max }+\mathrm{Q}_{\min }}{2} \times k
$$

where $\mathrm{Q}_{\max }$ and $\mathrm{Q}_{\min }$ are the maximum and the minimum quantization scale, respectively, and $k$ is a coefficient depending on the picture type. If the coding bit rate of the $n$th slice 
is in the range of

$$
\begin{aligned}
& \left(\frac{\text { Target Rate }}{\text { NO_slice } \times \text { Frame Rate }}\right) \times \mathrm{IR}_{\mathrm{H}} \\
& \quad \geq \text { slice }_{n}^{\mathrm{I}} \geq\left(\frac{\text { Target Rate }}{\text { NO_slice } \times \text { Frame Rate }}\right) \times \mathrm{IR}_{\mathrm{L}},
\end{aligned}
$$

where NO_slice is the number of slices in one frame, there will be no change in the quantization parameter. Otherwise, the quantization level is adjusted

$$
\begin{aligned}
& \text { if slice }{ }_{n}^{\mathrm{I}} \geq \frac{\mathrm{IR}_{\mathrm{H}} \times \text { Target Rate }}{\text { NO_slice } \times \text { Frame Rate }_{-}}, \quad \mathrm{Q}_{n+1}^{\mathrm{I}}=\mathrm{Q}_{n}^{\mathrm{I}}+1 ; \\
& \text { if slice }{ }_{n}^{\mathrm{I}} \leq \frac{\mathrm{IR}_{\mathrm{L}} \times \text { Target Rate }}{\text { NO_slice } \times \text { Frame Rate }}, \quad \mathrm{Q}_{n+1}^{\mathrm{I}}=\mathrm{Q}_{n}^{\mathrm{I}}-1 ;
\end{aligned}
$$

where $Q_{n}^{\mathrm{I}}$ and $\mathrm{Q}_{n+1}^{\mathrm{I}}$ denote the quantization scales for the current slice and the next slice, respectively. If the coding bit rate is over the predefined levels in the current slice, the quantization scale is increased or deceased by one level for the next slice in order to keep the specified bit rate. Hence, the coding rate can keep a dynamic balance during each frame coding. The final slice quantization scale is then recorded as an initial value for the first slice of the next I-frame coding.

In order to prevent the buffer from overflowing or underflowing, there should be a warning system for checking buffer status. In our method, the status of the buffer occupation is not frequently extracted for quantization adjustment. When the percentage of the buffer utilization $P_{0}$ falls in the range of $0.2 \leq P_{0} \leq 0.8$, the buffer operates in normal condition and the quantization level is not adjusted. Otherwise, the quantization level will be adjusted for the next slice coding as follows:

$$
\begin{array}{ll}
\text { if } P_{0} \geq 80 \%, & \mathrm{Q}_{n+1}^{\mathrm{I}}=\mathrm{Q}_{n}^{\mathrm{I}}+2 ; \\
\text { if } P_{0} \leq 20 \%, & \mathrm{Q}_{n+1}^{\mathrm{I}}=\mathrm{Q}_{n}^{\mathrm{I}}-2 ; \\
\text { others, } & \mathrm{Q}_{n+1}^{\mathrm{I}}=\mathrm{Q}_{n}^{\mathrm{I}} .
\end{array}
$$

From (10) and (11), the maximum quantization scale is increased by three when the slice coding rate is over the predefined level and the buffer utilization $P_{0} \geq 80 \%$. In another case, when the slice coding is lower than the predefined minimum level, but $P_{0} \geq 80 \%$, we also increase the quantization scale by one for the next slice coding.

Next, we discuss the rate control for P-frame coding. Because most of the temporal redundancy for P-frames can be removed by using motion compensations, the coding bit rate for the P-frame is not as high as that of an I-frame. The P-frame bit rate is then chosen close to the target bit rate with

$$
\frac{\text { Target Rate }}{\text { Frame Rate }} \times \mathrm{PR}_{\mathrm{H}} \geq \mathrm{P}_{\text {bit }} \geq \frac{\text { Target Rate }}{\text { Frame Rate }} \times \mathrm{PR}_{\mathrm{L}},
$$

where $\mathrm{PR}_{\mathrm{H}}$ and $\mathrm{PR}_{\mathrm{L}}$ denote the maximum and minimum control rates, respectively, and are usually close to unity. We also control the bit rate for P-frame coding with slice base, which can be expressed as

$$
\begin{aligned}
& \left(\frac{\text { Target Rate }}{\text { NO_slice } \times \text { Frame Rate }}\right) \times \mathrm{PR}_{\mathrm{H}} \\
& \quad \geq \text { slice }_{n}^{\mathrm{P}} \geq\left(\frac{\text { Target Rate }}{\text { NO_slice } \times \text { Frame Rate }}\right) \times \mathrm{PR}_{\mathrm{L}} .
\end{aligned}
$$

Similarly, to the I-frame coding, the quantization level for each slice of a P-frame is adaptively adjusted

$$
\begin{array}{ll}
\text { if slice }{ }_{n}^{\mathrm{P}} \geq \frac{\mathrm{PR}_{\mathrm{H}} \times \text { Target Rate }}{\text { NO_slice } \times \text { Frame Rate }}, & \mathrm{Q}_{n+1}^{\mathrm{P}}=\mathrm{Q}_{n}^{\mathrm{P}}+1 ; \\
\text { if slice }{ }_{n}^{\mathrm{P}} \leq \frac{\mathrm{IR}_{\mathrm{L}} \times \text { Target Rate }}{\text { NO_slice } \times \text { Frame Rate }}, & \mathrm{Q}_{n+1}^{\mathrm{P}}=\mathrm{Q}_{n}^{\mathrm{P}}-1 ; \\
\text { others, } & \mathrm{Q}_{n+1}^{\mathrm{P}}=\mathrm{Q}_{n}^{\mathrm{P}} .
\end{array}
$$

Hence, during one GOP coding, the total output bit rate is then

$$
\text { Output }_{\text {bit rate }}=\frac{\text { Target Rate } \times \text { NGOP }}{\text { Frame Rate }}
$$

where NGOP is the number of frames in one GOP. It is desirable to control the $\mathrm{GOP}_{\text {bit rate }}$ in (2), very close to the Output $_{\text {bit rate }}$, to obtain a dynamic balance in the entire GOP coding period. If the $\mathrm{GOP}_{\text {bit rate }}$ is equal to Output ${ }_{\text {bit rate, }}$ then

$$
\mathrm{I}_{\text {bit }}+3 \mathrm{P}_{\text {bit }}+8 \mathrm{~B}_{\text {bit }} \cong \frac{\text { Target Rate } \times 12}{\text { Frame Rate }}
$$

that is, the GOP structure is contained in one I-frame, three $\mathrm{P}$-frames, and eight B-frames, and thus we assume that all $\mathrm{P}$ and $\mathrm{B}$-frames have the same coding rate. In order to achieve the dynamic balance, the coding bit rates of $\mathrm{B}$-frames are adaptively modified to compensate for those of the I- and P-frames. Since B-frames are not used as references for motion prediction, the B-frame coding is not as important as that of the I-frame and P-frames. Moreover, B-frames use the bidirectional prediction, and so their coding errors will be smaller. From (9), (13), and (16), the B-frame bit rate is limited to

$$
\begin{aligned}
& \frac{\text { Target Rate }}{8 \times \text { Frame Rate }} \times\left(12-\mathrm{IR}_{\mathrm{L}}-3 \mathrm{PR}_{\mathrm{L}}\right) \\
& \quad \geq \mathrm{B}_{\text {bit }} \geq \frac{\text { Target Rate }}{8 \times \text { Frame Rate }} \times\left(12-\mathrm{IR}_{\mathrm{H}}-3 \mathrm{PR}_{\mathrm{H}}\right) .
\end{aligned}
$$

In order to control the B-frame bit rate, its quantization level is adjusted in each slice, which is similar to that of the Pframe coding. Meanwhile, the buffer occupation also must be periodically monitored during the $\mathrm{P}$ - and $\mathrm{B}$-frames coding, where the control procedure is the same as that of the I-frame coding. 


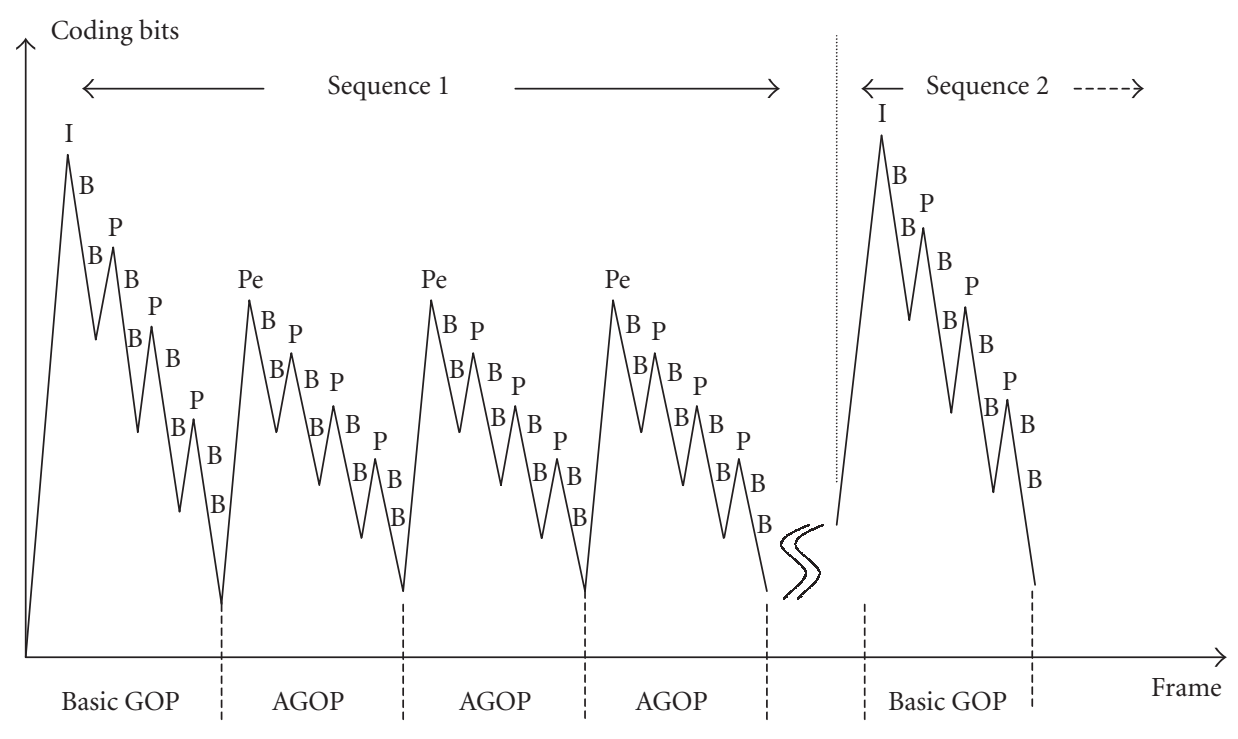

FIgUre 4: The ideal buffer occupation in the proposed adaptive GOP.

\subsection{For the AGOP}

In order to obtain higher coding efficiency, the use of intracoding in the same video sequence should be avoided if the temporal correlation is high, which can be done as follows. A video sequence can be partitioned into many AGOPs, and each AGOP consists of 12 frames as a coding unit that contains one enhanced P-frame $\left(\mathrm{P}_{e}\right)$, three P-frames, and eight $\mathrm{B}$-frames. The enhanced $\mathrm{P}$-frame is the starting point for each AGOP. Its position is like the I-frame of a BGOP, but its coding bit rate is not as high as an I-frame, which is given by

$$
\begin{aligned}
& \left(\frac{\text { Target Rate }}{\text { NO_slice } \times \text { Frame Rate }}\right) \times \mathrm{P}_{\mathrm{e}} \mathrm{R}_{\mathrm{H}} \\
& \quad \geq \text { slice }_{n}^{\mathrm{P}_{e}} \geq\left(\frac{\text { Target Rate }}{\text { NO_slice } \times \text { Frame Rate }}\right) \times \mathrm{P}_{\mathrm{e}} \mathrm{R}_{\mathrm{L}},
\end{aligned}
$$

where $\mathrm{PR}_{\mathrm{H}(\mathrm{L})}<\mathrm{P}_{\mathrm{e}} \mathrm{R}_{\mathrm{H}(\mathrm{L})}<\mathrm{IR}_{\mathrm{H}(\mathrm{L})}$. Its $\mathrm{P}$ - and $\mathrm{B}$-frame coding rates are similar to (12) and (17), respectively. The P- and B-coding bit rate may be slightly increased to improve the coding quality since the $\mathrm{P}_{\mathrm{e}}$-frame coding rate is usually less than that of the I-frame. The coding performance of the entire video sequence is then greatly improved from the motion compensation. The ideal buffer occupation of the proposed AGOP method is illustrated in Figure 4, where the coding bit rate can maintain dynamic balance during the entire GOP coding. However, coding bit rates can vary drastically for different video sequences, so it is not easy to achieve an ideal buffer occupation for each GOP coding. Hence, we need to monitor the buffer status at the end of each GOP. If the buffer is occupied by one half or more at the end of the GOP coding, the coding rate should be decreased in the next GOP to achieve the coding bit rate balance.

\section{EXPERIMENTAL SIMULATIONS}

In order to test the performance of our algorithm, four video sequences "Football," "Susie," "Flower-garden," and "Salesman," the frame size with $352 \times 288$ resolutions, were employed. To simulate the practical video sequences, we pasted the parts of each sequence together to form a test sequence as follows. The first 1-50 frames are from the "Football," the 51-100 frames are from the "Phone-lady," the 101-150 frames are from the "Flower-garden," and finally the 151-200 frames are from the "Salesman." For comparisons, we also tested this sequence using the well-known TM5 method [11].

The simulations were done under the condition of $400 \mathrm{k}$-bit buffer size, $1.2 \mathrm{M}$ target bit rate, 30 frames per second, and the range of the motion search was $-16 \sim+16$. The initial parameters were set at $\mathrm{IR}_{\mathrm{H}}=5, \mathrm{IR}_{\mathrm{L}}=4.5, \mathrm{PR}_{\mathrm{H}}=1.5$, and $\mathrm{PR}_{\mathrm{L}}=1.2$ for BGOP; and $\mathrm{P}_{\mathrm{e}} \mathrm{R}_{\mathrm{H}}=4, \mathrm{P}_{\mathrm{e}} \mathrm{R}_{\mathrm{L}}=3.5$, $\mathrm{PR}_{\mathrm{H}}=1.7$, and $\mathrm{PR}_{\mathrm{L}}=1.4$ for AGOP. These parameters may have $\pm 10 \%$ adjustments according to the buffer status. Figure 5 a shows the result of coding bit in each frame. In our scheme, the averaged bit rates of I- and P-frames are larger than that of the TM5 to reduce the predicted errors; and our bit rate of the B-frame is less than that of the TM5 to obtain the coding bit rate balance. Next, we compared the buffer status, and the results are shown in Figure 5b. In the TM5 method, the bit allocation is not exact for each frame coding, hence the buffer underflowed during the 158th-165th frames. In our coding method, since the utility ratio of buffer is always forced to settle in the range of $80 \% \sim 20 \%$ occupation, no underflow or overflow occurred. At the high motion sequences such as "Football" and "Flower-garden," at times the buffer exceeds the utility ratio, but we can prevent the buffer from overflowing since there is $20 \%$ reservation. As the coding bit rate becomes very high, the quantization level 


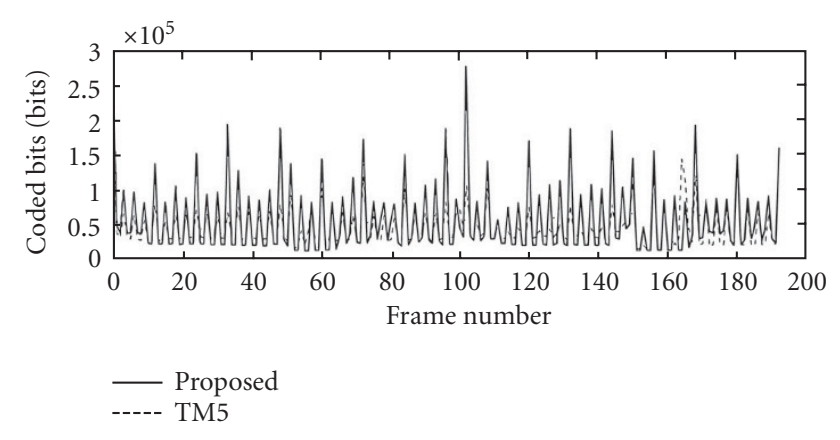

(a) The coding rate for each frame.

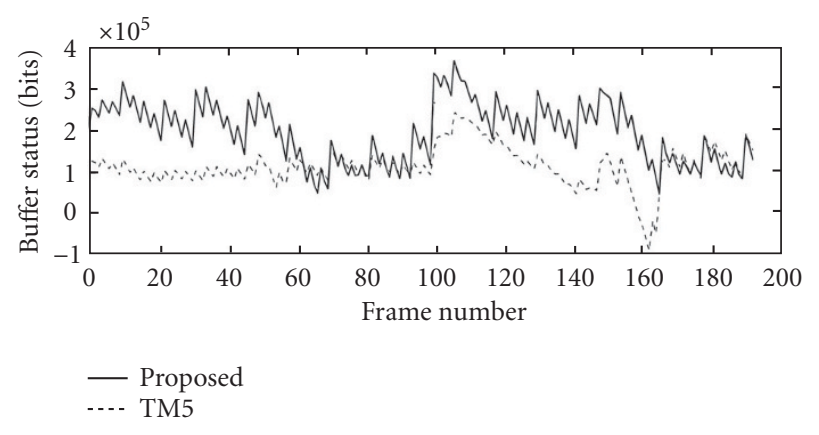

(b) The buffer occupation during 200 frames coding.

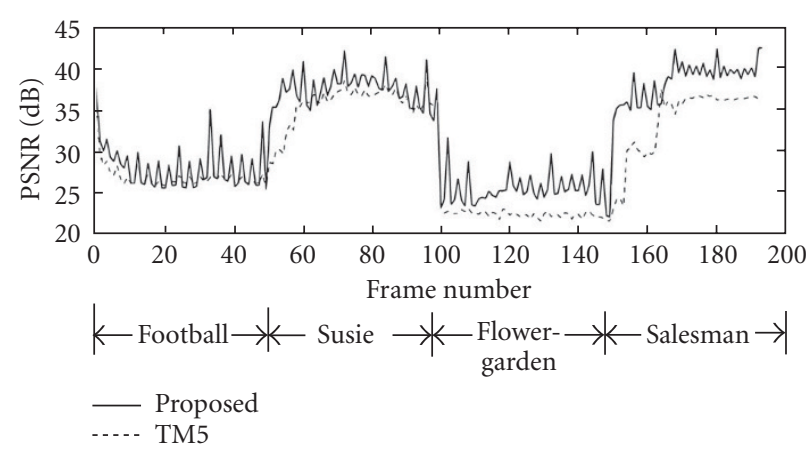

(c) The coding quality estimation for each frame.

FIGURE 5

was gradually increased for the next slice coding in order to avoid degradations of the coding quality suddenly. The buffer occupation was then slowly decreased as the coding continues. During the 200 frames coding, the final buffer occupation in our method is almost the same as that of the TM5, and the coding rate of our method was able to keep balance throughout the entire processing.

Next, we measured our coding quality using the above parameters with the results as shown in Figure 5c. Our adaptive algorithm achieved an improvement of about $2 \sim 5 \mathrm{~dB}$ PSNR on the average compared with the TM5 method for various sequences. The results show that our algorithm can provide much better quality for low motion sequences such as "Salesman." We also notice the performance of the

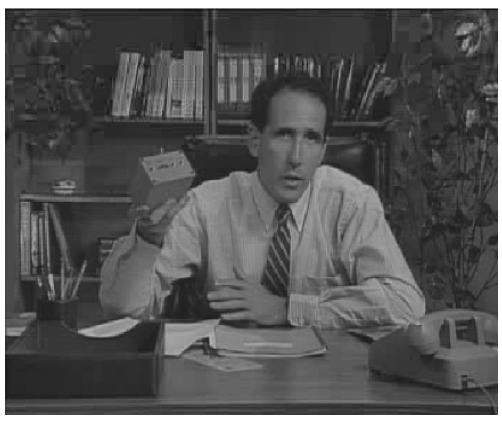

(a) The decoded 151th image with our proposed method $($ PSNR $=35.09 \mathrm{~dB})$.

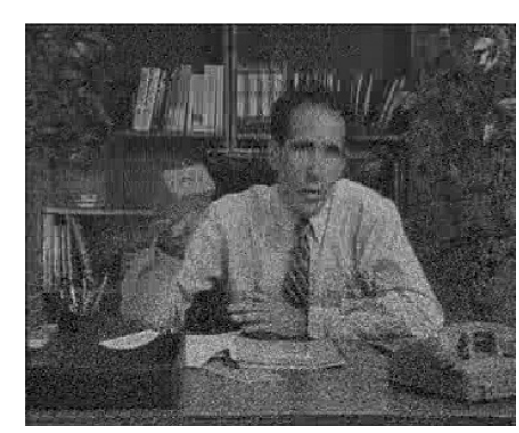

(b) The decoded 151th image with TM5 method $($ PSNR $=23.78 \mathrm{~dB})$.

Figure 6

decoding sequence at the scene change. The decoded frames are individually shown in Figure 6 using our algorithm and the TM5 coding at the 151th frame. The TM5 method usually produces serious distortions in the decoded image due to high predicted errors at the scene change, but no visible distortion was found from the reconstructed image by our method. Moreover, we compare the coding quality between the I-frame of the static GOP using TM5 method and the enhanced P-frame of the proposed AGOP. Figure 7 shows our enhanced P-frame and the decoded I-frame result at the 180th frame. Clearly, the proposed rate control scheme can improve the coding efficiency.

The coding performance is dependent on the reliability of scene change. To test and compare the function of scene change, two completing algorithms for scene change were evaluated $[13,14]$. We simulated two programs "Top Gun," and "Weather Forecast." To evaluate the detection performance of the scene change, we define a testing parameter as

$$
\text { Reliability }=\frac{N c-N f}{N c+N m} \times 100 \%,
$$

where $N c$ is the number of correct detection, $N m$ is the number of missed detections, and $N f$ is the number of false detection. In the "Top gun" program, there are 7630 frames, which have 156 scene changes. Another "Weather Forecast" program uses 6760 frames, with 48 scene changes. 
TABLE 1 Comparisons of scene change detection performance.

\begin{tabular}{|c|c|c|c|c|}
\hline Sequences & Methods & Kang et al. [13] & Huang et al. [14] & Proposed \\
\hline Top Gun (7630 frames) & $\begin{array}{l}\text { Correct detection } \\
\text { False detection } \\
\text { Missed detection } \\
\text { Reliability }\end{array}$ & $\begin{array}{c}149 \\
6 \\
7 \\
92 \%\end{array}$ & $\begin{array}{c}150 \\
4 \\
6 \\
94 \%\end{array}$ & $\begin{array}{c}151 \\
9 \\
5 \\
92 \%\end{array}$ \\
\hline Weather Forecast (6760 frames) & $\begin{array}{l}\text { Correct detection } \\
\text { False detection } \\
\text { Missed detection } \\
\text { Reliability }\end{array}$ & $\begin{array}{c}46 \\
2 \\
2 \\
92 \%\end{array}$ & $\begin{array}{c}47 \\
1 \\
1 \\
96 \%\end{array}$ & $\begin{array}{c}47 \\
3 \\
1 \\
92 \%\end{array}$ \\
\hline
\end{tabular}

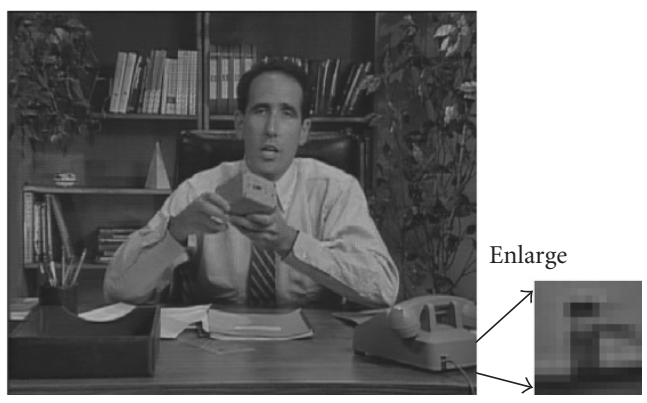

(a) The decoded 180th image with our proposed method $($ PSNR $=42.55 \mathrm{~dB})$.

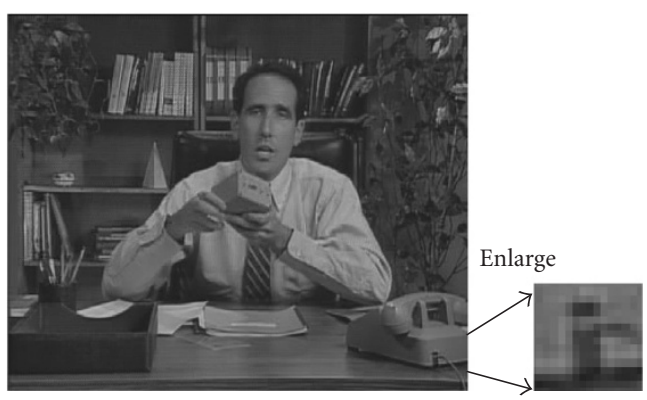

(b) The decoded 180th image with TM5 method $($ PSNR $=36.57 \mathrm{~dB})$.

FIgURE 7

The former has much higher motion and more scene changes than the later. The results are listed in Table 1. Simulations demonstrate that our scene change detection can achieve about $92 \%$ reliability, which is close to the other highperformance algorithms $[13,14]$. For practical video encoding applications, the number of missed detections should be as low as possible since the coding quality degrades seriously if the scene change point cannot be found. So we can reduce the detection threshold in (4). However, the number of false detections would be increased, and the length of AGOP is shortened accordingly. In the worst case, our performance is the same as static GOP since the minimum length of AGOP is set with 12 frames. This is acceptable for practical coding systems since I-mode coding only increases the coding bit rate but without serious prediction errors. Moreover, our scene detection method only extracts the coding parameters, that is, slice coding rate and quantization scale, from the video encoder and adopts a simple analysis to find the scene change. Hence, the computational complexity of the proposed scene change detection is clearly lower than that of the other methods.

\section{CONCLUSIONS}

In this study, we proposed a novel video coding control algorithm by using an AGOP approach instead of the static GOP structure. The current temporal correlation between the two neighboring frames is monitored and used for BGOP/AGOP switching decision with low computational load to make it applicable to real-time systems. This is basically done by using the expensive intramode coding, only if a scene change is detected or the temporal correlation becomes low. An Ipicture is adaptively replaced by an enhanced P-picture to improve the coding efficiency. The slice-based coding control scheme is used to satisfy the real-time coding requirements and to avoid re-encoding even if a scene change is found. Simulations demonstrated that the proposed method achieves better results than the TM5 model and provides enough accuracy to detect scene changes.

\section{ACKNOWLEDGMENTS}

The author acknowledges the suggestions made by the anonymous reviewers for improving the paper, and thanks the National Science Council, Taiwan, (NSC90-2213-E-327010) for supporting this research, and thanks Chung-Long Chen for simulating partial algorithms.

\section{REFERENCES}

[1] M. Liou, "Overview of the $p \times 64 \mathrm{kbits} / \mathrm{s}$ video coding standard," Communications of the ACM, vol. 34, no. 4, pp. 59-63, 1991.

[2] MPEG-2 video, ISO/IEC DIS 13818-2.

[3] G. Cote, B. Erol, M. Gallant, and F. Kossentini, "H.263+: video coding at low bit-rate," IEEE Trans. Circuits and Systems for Video Technology, vol. 8, no. 7, pp. 849-866, 1998. 
[4] C. F. Chang and J. S. Wang, "A stable buffer control strategy for MPEG coding," IEEE Trans. Circuits and Systems for Video Technology, vol. 7, no. 6, pp. 920-924, 1997.

[5] L. Wang and A. Vincent, "Joint rate control for multiprogram video coding," IEEE Trans. Consumer Electronics, vol. 42, no. 3, pp. 300-305, 1996.

[6] M. R. Pickering and J. F. Arnold, "A perceptually efficient VBR rate control algorithm," IEEE Trans. Image Processing, vol. 3, no. 5, pp. 527-532, 1996.

[7] J. Lee and B. W. Dickinson, "Rate-distortion optimized frame type selection for MPEG encoding," IEEE Trans. Circuits and Systems for Video Technology, vol. 7, no. 3, pp. 501-509, 1997.

[8] S. W. Wu and A. Gersho, "Rate-constrained optimal blockadaptive coding for digital tape recording of HDTV," IEEE Trans. Circuits and Systems for Video Technology, vol. 1, no. 1, pp. 100-112, 1991.

[9] H. Sun, W. Kwok, M. Chien, and C. H. John, "MPEG coding performance improvement by jointly optimizing coding mode decision and rate control," IEEE Trans. Circuits and Systems for Video Technology, vol. 7, no. 3, pp. 449-458, 1997.

[10] T. Chiang and Y.-Q. Zhang, "A new rate control scheme using quadratic rate distortion model," IEEE Trans. Circuits and Systems for Video Technology, vol. 7, no. 1, pp. 246-250, 1997.

[11] ISO/IEC-JTC1/SC29/WG11: Test Model 5, MPEG93/N0400, 1993.

[12] H. Gharavi and M. Mills, "Block-matching motion estimation algorithms-new results," IEEE Trans. Circuits and Systems, vol. 37, pp. 649-651, 1997.

[13] E. K. Kang, S. J. Kim, and J. S. Choi, "Video retrieval based on scene change detection in compressed streams," IEEE Trans. Consumer Electronics, vol. 45, no. 3, pp. 932-936, 1999.

[14] C. L. Huang and B. Y. Liao, "A robust scene-change detection method for video segmentation," IEEE Trans. Circuits and Systems for Video Technology, vol. 11, no. 12, pp. 1281$1288,2001$.

Shih-Chang Hsia was born in Yuanlin, Taiwan, in 1962 . He received the Ph.D. degree from the Department of Electrical Engineering, National Cheng Kung University, T'ai-nan, Taiwan, in 1997. During 19861989, he was an Engineer in the R\&D Department of Microtek International, Inc., Hsin-Chu. He was an Instructor and Associate Professor in the Department of electronic engineering, Chung Chou Institute

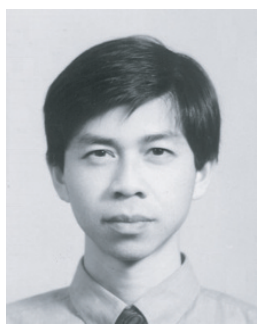
of Technology, during 1991-1998. Currently, he is an Associate Professor in the Department of Computer and Communication Engineering, National Kaohsiung First University of Science and Technology Kaohsiung. His research interests include VLSI design, HDTV and cable systems, video coding and processing, communication, and data hiding systems. 